\title{
Accuracy-enhancing methods for balancing-related frequency-weighted model and controller reduction ${ }^{2}$
}

\author{
Andras Varga ${ }^{\mathrm{a}, *}$, Brian D.O. Anderson ${ }^{\mathrm{b}}$ \\ ${ }^{a}$ German Aerospace Center, DLR-Oberpfaffenhofen, Institute of Robotics and Mechatronics, D-82234 Wessling, Germany \\ ${ }^{\mathrm{b}}$ Research School of Information Sciences and Engineering, Australian National University, Canberra ACT 0200, Australia
}

Received 28 December 2001; received in revised form 20 December 2002; accepted 21 January 2003

\begin{abstract}
We consider the solution of the balancing-related frequency-weighted model and controller reduction problems using accuracy enhanced numerical algorithms. We propose first new stability-enforcing choices of the frequency-weighted grammians which can guarantee the stability of reduced models for two-sided frequency weights. Then we show that for the frequency-weighted controller reduction problems with standard stability and performance-enforcing frequency weights the computation of the frequency-weighted grammians can be done by solving reduced order Lyapunov equations. For both frequency-weighted model and controller reduction problems we indicate how to compute the grammians directly in terms of their Cholesky factors. This allows the extension of the square-root and balancing-free accuracy-enhancing techniques to the frequency-weighted case.
\end{abstract}

(C) 2003 Elsevier Science Ltd. All rights reserved.

Keywords: Model reduction; Balancing; Frequency-weighting; Controller reduction; Numerical algorithms

\section{Introduction}

Consider the $n$th order original continuous-time state-space model $\mathbf{G}:=(A, B, C, D)$ with the transfer-function matrix (TFM)

$G(s)=C(s I-A)^{-1} B+D$

and let $\mathbf{G}_{r}:=\left(A_{r}, B_{r}, C_{r}, D_{r}\right)$ be an $r$ th order approximation of the original model $(r<n)$, with the TFM

$G_{r}(s)=C_{r}\left(s I-A_{r}\right)^{-1} B_{r}+D_{r}$.

The methods for frequency-weighted model reduction (FWMR) try to minimize a weighted approximation error of the form

$\left\|W_{\mathrm{o}}\left(G-G_{r}\right) W_{\mathrm{i}}\right\|_{\infty}$,

\footnotetext{
Part of this paper was presented at the 15th IFAC World Congress in Barcelona, July 21-26, 2002. This paper was recommended for publication in revised form by Editor Roberto Tempo.

* Corresponding author. Tel.: +49-8153-282407; fax: +49-8153281441.

E-mail addresses: andras.varga@dlr.de (A. Varga), brian.anderson@ anu.edu.au (B.D.O. Anderson).
}

where $W_{\mathrm{o}}$ and $W_{\mathrm{i}}$ are suitably chosen output and input weighting TFMs, respectively.

Notation: Throughout the paper, we will use the bold-notation $\mathbf{G}$ to denote a state-space system having the TFM $G(s)$ or $G$. This notation is also used to denote state-space realizations of systems corresponding to operations with TFMs, as for example, $\mathbf{G}_{1} \mathbf{G}_{2}$ to denote the series coupling of two systems having the TFM $G_{1}(s) G_{2}(s)$ or $\mathbf{G}_{1}+\mathbf{G}_{2}$ to denote the (additive) parallel coupling of two systems with TFM $G_{1}(s)+G_{2}(s)$.

Controller reduction problems are frequently formulated as FWMR problems (Anderson \& Liu, 1989). Let $\mathbf{K}=$ $\left(A_{\mathrm{c}}, B_{\mathrm{c}}, C_{\mathrm{c}}, D_{\mathrm{c}}\right)$ be a stabilizing controller of order $n_{\mathrm{c}}$ for the system $\mathbf{G}$. We want to find $\mathbf{K}_{r}$, an $r_{\mathrm{c}}$ th order approximation of $\mathbf{K}$ having the same number of unstable poles as $\mathbf{K}$, such that a weighted error of the form

$$
\left\|W_{\mathrm{o}}\left(K-K_{r}\right) W_{\mathrm{i}}\right\|_{\infty}
$$

is minimized. To enforce closed-loop stability one-sided weights of the form

$$
\begin{aligned}
& W_{\mathrm{o}}=(I+G K)^{-1} G, \quad W_{\mathrm{i}}=I \\
& W_{\mathrm{o}}=I, \quad W_{\mathrm{i}}=G(I+K G)^{-1}
\end{aligned}
$$


can be used, while performance-preserving considerations lead to two-sided weights

$$
W_{\mathrm{o}}=(I+G K)^{-1} G, \quad W_{\mathrm{i}}=(I+G K)^{-1} .
$$

To solve the approximation problem of minimizing the weighted error norm (1), a possible suboptimal but easy to apply approach has been proposed by Enns (1984). This approach is summarized below:

\section{FWMR procedure}

1. Compute an additive stable-unstable spectral decomposition of $\mathbf{G}$ as $\mathbf{G}=\mathbf{G}_{\mathrm{s}}+\mathbf{G}_{\mathrm{u}}$, where $\mathbf{G}_{\mathrm{s}}$, of order $n_{\mathrm{s}}$, contains the stable poles of $\mathbf{G}$ and $\mathbf{G}_{\mathrm{u}}$, of order $n-n_{\mathrm{s}}$, contains the unstable poles of $\mathbf{G}$.

2. Compute the controllability grammian of $\mathbf{G}_{\mathrm{s}} \mathbf{W}_{\mathrm{i}}$ and the observability grammian of $\mathbf{W}_{\mathrm{o}} \mathbf{G}_{\mathrm{s}}$ and define, according to Enns (1984) (see Section 2), appropriate $n_{\mathrm{s}}$ order frequency-weighted controllability and observability grammians $P_{\mathrm{E}}$ and $Q_{\mathrm{E}}$, respectively.

3. Using $P_{\mathrm{E}}$ and $Q_{\mathrm{E}}$ in place of standard grammians of $\mathbf{G}_{\mathrm{s}}$, determine a reduced order approximation $\mathbf{G}_{s r}$ by applying the balanced truncation (BT) approach of Moore (1981).

4. Compute $\mathbf{G}_{r}=\mathbf{G}_{\mathrm{s} r}+\mathbf{G}_{\mathrm{u}}$.

In this paper we discuss several enhancements of Enns' approach. A first possibility which we only mention in passing, is to use the singular perturbation approximation (SPA) method (Liu \& Anderson, 1989) instead of BT at step 3 of the FWMR procedure. As revealed by several examples given in (Varga \& Anderson, 2001) (see also Section 5 ), the SPA used in conjunction with frequency-weighted balancing techniques exhibits superior performance over the more traditionally used BT method, both in preserving stability as well as in providing better approximations.

Further enhancing of Enns' method tries to remedy the main difficulty with this method, namely, the lack of guarantee of stability of the reduced model in the case of two-sided weighting. In Section 2 we propose new parameterized selection schemes of the grammians which combine the advantages of Enns' method with those of the stability guaranteeing techniques proposed by Lin and Chiu (1992) and Wang, Sreeram, and Liu (1999). The numerical example in Section 5 illustrates the additional flexibility provided by the new selection schemes.

In Section 3 we discuss enhancements of Enns' approach when applied to the controller reduction problem (2) for the special weighs defined in (3)-(5). The main result shows that for an arbitrary stabilizing controller, the computation of grammians can be efficiently performed by solving Lyapunov equations of order at most $n+n_{\mathrm{c}}$ (instead the expected order of $n+2 n_{\mathrm{c}}$ ). This result and its corollary extend similar results of Schelfhout and De Moor (1996), established for stable controllers and strictly proper plants, and of Liu, Anderson, and Ly (1990), stated for stable state-feedback and full-order estimator-based controllers, respectively.

Reliable numerical methods for unweighted approximations are well understood and accompanying robust numerical software based on balancing-free square-root accuracy-enhancing techniques (Varga, 1991a,b) is freely available (Varga, 2001). In Section 4 we extend these techniques to the balancing-related frequency-weighted approximation method. Specifically, we develop formulas to compute the Cholesky (square-root) factors of the frequency-weighted grammians for all proposed choices in Sections 2 and 3.

\section{Balancing-related frequency-weighted model reduction}

\subsection{Review of known techniques}

Balancing-related model reduction can be interpreted as performing a similarity transformation $Z$ on a stable original system $\mathbf{G}=(A, B, C, D)$ yielding

$$
\left[\begin{array}{c|c}
Z^{-1} A Z & Z^{-1} B \\
\hline C Z & D
\end{array}\right]:=\left[\begin{array}{cc|c}
A_{11} & A_{12} & B_{1} \\
A_{21} & A_{22} & B_{2} \\
\hline C_{1} & C_{2} & D
\end{array}\right]
$$

and then defining the reduced model of order $r$ as $\mathbf{G}_{r}=$ $\left(A_{11}, B_{1}, C_{1}, D\right)$. When partitioning compatibly $Z$ and $Z^{-1}$ in the form

$Z:=\left[\begin{array}{ll}T & U\end{array}\right], \quad Z^{-1}:=\left[\begin{array}{l}L \\ V\end{array}\right]$,

then $\Pi=T L$ is a projector on $T$ along $L$, and $L T=I_{r}$. Thus the reduced system is given by

$\mathbf{G}_{r}=(L A T, L B, C T, D)$.

The matrices $L$ and $T$, called truncation matrices, are the only ones necessary to determine the matrices of the reduced model $\mathbf{G}_{r}$.

Partitioned representations as in (6) can also be used to construct a SPA $\mathbf{G}_{r}$ of $\mathbf{G}$ by employing the SPA formulas

$$
\left[\begin{array}{c|c}
A_{r} & B_{r} \\
\hline C_{r} & D_{r}
\end{array}\right]=\left[\begin{array}{c|c}
A_{11}-A_{12} A_{22}^{-1} A_{21} & B_{1}-A_{12} A_{22}^{-1} B_{2} \\
\hline C_{1}-C_{2} A_{22}^{-1} A_{21} & D-C_{2} A_{22}^{-1} B_{2}
\end{array}\right] .
$$

Note that the SPA method preserves the DC-gain of an original stable system.

For balancing-related model reduction methods, the computation of suitable truncation matrices relies on two positive semidefinite symmetric matrices $P$ and $Q$, called generically the controllability and observability grammians, respectively. For the unweighted BT and SPA methods applied to a system G (Moore, 1981; Liu \& Anderson, 1989), 
$P$ and $Q$ satisfy a pair of Lyapunov equations:

$$
\begin{aligned}
& A P+P A^{\mathrm{T}}+B B^{\mathrm{T}}=0, \\
& A^{\mathrm{T}} Q+Q A+C^{\mathrm{T}} C=0 .
\end{aligned}
$$

Since the grammians of a stable system are positive semidefinite matrices, the stability of the reduced models follows from the guaranteed positive semidefiniteness of their grammians.

The grammians can be always determined in Cholesky factorized forms $P=S S^{\mathrm{T}}$ and $Q=R^{\mathrm{T}} R$, where $S$ and $R$ are upper-triangular matrices. The computation of the truncation matrices $L$ and $T$ can be done from the singular value decomposition (SVD)

$R S=\left[\begin{array}{ll}U_{1} & U_{2}\end{array}\right] \operatorname{diag}\left(\Sigma_{1}, \Sigma_{2}\right)\left[\begin{array}{ll}V_{1} & V_{2}\end{array}\right]^{\mathrm{T}}$,

where $\Sigma_{1}=\operatorname{diag}\left(\sigma_{1}, \ldots, \sigma_{r}\right), \Sigma_{2}=\operatorname{diag}\left(\sigma_{r+1}, \ldots, \sigma_{n}\right)$, and $\sigma_{1} \geqslant \cdots \geqslant \sigma_{r}>\sigma_{r+1} \geqslant \cdots \geqslant \sigma_{n} \geqslant 0$. Note that $\sigma_{1}, \ldots, \sigma_{n}$ are the Hankel-singular values of the system $\mathbf{G}$. To compute $L$ and $T$, the formulas

$L=\Sigma_{1}^{-1 / 2} U_{1}^{\mathrm{T}} R, \quad T=S V_{1} \Sigma_{1}^{-1 / 2}$

proposed by Tombs and Postlethwaite (1987) can be used. This is the so-called square-root method to compute the truncation matrices, and its name suggests that all computations are done in terms of square-root quantities (i.e., the Cholesky factors).

The frequency-weighted balanced truncation (FWBT) and frequency-weighted singular perturbation approximation (FWSPA) methods extend the unweighted BT and SPA methods to the frequency-weighted case and rely on so-called frequency-weighted grammians. The first FWBT approach has been proposed by Enns (1984). From now, let assume $G$ and the two weights $W_{\mathrm{o}}$ and $W_{\mathrm{i}}$ are all stable TFMs, and let $\mathbf{W}_{\mathrm{o}}=\left(A_{\mathrm{o}}, B_{\mathrm{o}}, C_{\mathrm{o}}, D_{\mathrm{o}}\right)$ and $\mathbf{W}_{\mathrm{i}}=\left(A_{\mathrm{i}}, B_{\mathrm{i}}, C_{\mathrm{i}}, D_{\mathrm{i}}\right)$ be minimal state-space realizations of the weighting matrices. Consider the following realizations of $\mathbf{G} \mathbf{W}_{\mathrm{i}}$ and $\mathbf{W}_{\mathrm{o}} \mathbf{G}$ :

$$
\begin{aligned}
& \mathbf{G W}_{\mathrm{i}}=\left[\begin{array}{c|c}
\bar{A}_{\mathrm{i}} & \bar{B}_{\mathrm{i}} \\
\hline \bar{C}_{\mathrm{i}} & \bar{D}_{\mathrm{i}}
\end{array}\right]=:\left[\begin{array}{cc|c}
A & B C_{\mathrm{i}} & B D_{\mathrm{i}} \\
0 & A_{\mathrm{i}} & B_{\mathrm{i}} \\
\hline C & D C_{\mathrm{i}} & D D_{\mathrm{i}}
\end{array}\right], \\
& \mathbf{W}_{\mathrm{o}} \mathbf{G}=\left[\begin{array}{c|c}
\bar{A}_{\mathrm{o}} & \bar{B}_{\mathrm{o}} \\
\hline \bar{C}_{\mathrm{o}} & \bar{D}_{\mathrm{o}}
\end{array}\right]=:\left[\begin{array}{cc|c}
A_{\mathrm{o}} & B_{\mathrm{o}} C & B_{\mathrm{o}} D \\
0 & A & B \\
\hline C_{\mathrm{o}} & D_{\mathrm{o}} C & D_{\mathrm{o}} D
\end{array}\right] .
\end{aligned}
$$

Let

$\bar{P}=\left[\begin{array}{ll}P_{11} & P_{12} \\ P_{12}^{\mathrm{T}} & P_{22}\end{array}\right], \quad \bar{Q}=\left[\begin{array}{ll}Q_{11} & Q_{12} \\ Q_{12}^{\mathrm{T}} & Q_{22}\end{array}\right]$,

be the controllability grammian of $\mathbf{G} \mathbf{W}_{\mathrm{i}}$ and the observability grammian of $\mathbf{W}_{\mathrm{o}} \mathbf{G}$, respectively, partitioned such that $P_{11}$ and $Q_{22}$ are $n \times n$ matrices. The approach proposed by Enns defines

$P_{\mathrm{E}}=P_{11}, \quad Q_{\mathrm{E}}=Q_{22}$ as the frequency-weighted controllability and observability grammians, respectively. Although this method has been successfully employed in many applications, its main weakness is the lack of guaranteed stability of the reduced model in the case of two-sided weighting $\left(W_{\mathrm{o}} \neq I\right.$ and $\left.W_{\mathrm{i}} \neq I\right)$ (Anderson \& Liu, 1989).

An alternative approach was proposed by Lin and Chiu (1992) which, under certain assumptions, guarantees stability in the case of two-sided weighting. Provided $P_{22}$ and $Q_{11}$ are nonsingular (condition ensured if $\mathbf{W}_{\mathrm{i}}$ and $\mathbf{W}_{\mathrm{o}}$ are minimal realizations), the frequency-weighted grammians are chosen as

$$
\begin{aligned}
& P_{\mathrm{L}}=P_{11}-P_{12} P_{22}^{-1} P_{12}^{\mathrm{T}}, \\
& Q_{\mathrm{L}}=Q_{22}-Q_{12}^{\mathrm{T}} Q_{11}^{-1} Q_{12} .
\end{aligned}
$$

Since $P_{\mathrm{L}}$ and $Q_{\mathrm{L}}$ satisfy this time Lyapunov equations of the form (10) (however with different $B$ and $C$ matrices), the stability of the reduced model is automatically guaranteed (Pernebo \& Silverman, 1982). The main weakness of this approach is the requirement that no pole-zero cancellations occur when forming $\mathbf{G W}_{\mathrm{i}}$ or $\mathbf{W}_{\mathrm{o}} \mathbf{G}$. For example, this restriction prevents the applicability of this method to solve controller reduction problems involving weights as in (3), (4), or (5), where pole-zero cancellation takes always place (sea also Section 3 ).

Another modification of the method of Enns which guarantees stability in the case of two-sided weighting has been proposed by Wang et al. (1999) for continuous-time systems. Two frequency-weighted grammians $P_{\mathrm{W}}$ and $Q_{\mathrm{W}}$ are determined as the solutions of the pair of Lyapunov equations

$$
\begin{aligned}
& A P_{\mathrm{W}}+P_{\mathrm{W}} A^{\mathrm{T}}+\tilde{B} \tilde{B}^{\mathrm{T}}=0, \\
& Q_{\mathrm{W}} A+A^{\mathrm{T}} Q_{\mathrm{W}}+\tilde{C}^{\mathrm{T}} \tilde{C}=0,
\end{aligned}
$$

where $\tilde{B}$ and $\tilde{C}$ are fictitious input and output matrices. These matrices are computed from the orthogonal eigendecompositions of the symmetric matrices $X=-A P_{\mathrm{E}}-P_{\mathrm{E}} A^{\mathrm{T}}$ and $Y=-A^{\mathrm{T}} Q_{\mathrm{E}}-Q_{\mathrm{E}} A$ :

$X=U \Theta U^{\mathrm{T}}, \quad Y=V \Gamma V^{\mathrm{T}}$,

where $\Theta$ and $\Gamma$ are real diagonal matrices. Specifically, $\tilde{B}$ and $\tilde{C}$ are determined as

$\tilde{B}=U|\Theta|^{1 / 2}, \quad \tilde{C}=|\Gamma|^{1 / 2} V^{\mathrm{T}}$,

where $|\cdot|$ denotes a matrix formed from the absolute values of its elements. It is easy to see that with this choice of grammians we have $P_{\mathrm{W}}-P_{\mathrm{E}}=: \Delta P_{\mathrm{W}} \geqslant 0$ and $Q_{\mathrm{W}}-Q_{\mathrm{E}}=$ : $\Delta Q_{\mathrm{W}} \geqslant 0$. Thus, the system $(A, \tilde{B}, \tilde{C})$ is minimal provided the original system is minimal.

\subsection{New developments}

It is possible to construct frequency-weighted grammians which lead to a combination of the approach of Lin and Chiu 
(1992) with Enns' method. In such a combination method we can use as frequency-weighted grammians

$$
\begin{aligned}
& P_{\mathrm{EL}}=P_{11}-\alpha_{\mathrm{c}}^{2} P_{12} P_{22}^{-1} P_{12}^{\mathrm{T}}, \\
& Q_{\mathrm{EL}}=Q_{22}-\alpha_{\mathrm{o}}^{2} Q_{12}^{\mathrm{T}} Q_{11}^{-1} Q_{12},
\end{aligned}
$$

where for $\alpha_{\mathrm{c}}=\alpha_{\mathrm{o}}=0$ we have the choice for Enns' method, while for $\alpha_{c}=\alpha_{o}=1$ we have the choice for the method of Lin and Chiu (1992) with stability guarantee. Because stability is guaranteed for $\alpha_{c}=\alpha_{o}=1$, it is to be expected this to be also true for nearby subunitary values of $\alpha_{c}$ and $\alpha_{\mathrm{o}}$ (on the basis of a continuous variation of spectrum with $\alpha_{c}$ and $\alpha_{0}$ ). Thus, stability will be guaranteed in a whole neighborhood of $\alpha_{c}=\alpha_{0}=1$ regardless of whether pole-zero cancellations occur or not. This feature can be seen as a simultaneous enhancement of both methods.

Concerning enhancements of the approach of Wang et al. (1999), it appears that in some cases the distance to Enns' choice (i.e., the sizes of $\Delta P_{\mathrm{W}}$ and $\Delta Q_{\mathrm{W}}$ ) can be reduced by another choice of $\tilde{B}$ and $\tilde{C}$. Consider $\Theta=\operatorname{diag}\left(\Theta_{1}, \Theta_{2}\right)$ and $\Gamma=\operatorname{diag}\left(\Gamma_{1}, \Gamma_{2}\right)$ in the decompositions of $X$ and $Y$ in (18) partitioned such that $\Theta_{1}>0$ and $\Theta_{2} \leqslant 0, \Gamma_{1}>0$ and $\Gamma_{2} \leqslant 0$. Partition $U=\left[\begin{array}{ll}U_{1} & U_{2}\end{array}\right]$ and $V=\left[\begin{array}{ll}V_{1} & V_{2}\end{array}\right]$ in accordance with the partitioning of $\Theta$ and $\Gamma$, respectively, and define

$\tilde{B}=U_{1} \Theta_{1}^{1 / 2}, \quad \tilde{C}=\Gamma_{1}^{1 / 2} V_{1}^{\mathrm{T}}$.

With this choice, an a priori weighted approximation error bound as in (Wang et al., 1999) holds, provided some rank conditions are additionally fulfilled. This modification of the method of Wang et al. (1999) appears to be useful in some applications (see example in Section 5).

It is possible now to consider a modified combination method, where we include all above modifications in a single parameterized approach, which guarantees the stability of reduced models for two-sided weighting. This can be done by defining new fictitious input and output matrices $\hat{B}$ and $\hat{C}$, respectively, such that the frequency-weighted grammians $P_{\mathrm{V}}$ and $Q_{\mathrm{V}}$ satisfy

$$
\begin{aligned}
& A P_{\mathrm{V}}+P_{\mathrm{V}} A^{\mathrm{T}}+\hat{B} \hat{B}^{\mathrm{T}}=0, \\
& Q_{\mathrm{V}} A+A^{\mathrm{T}} Q_{\mathrm{V}}+\hat{C}^{\mathrm{T}} \hat{C}=0,
\end{aligned}
$$

$\hat{B}$ and $\hat{C}$ are defined just like $\tilde{B}$ and $\tilde{C}$ in (20), using the decompositions (18) but with $X=-A P_{\mathrm{EL}}-P_{\mathrm{EL}} A^{\mathrm{T}}$ and $Y=-A^{\mathrm{T}} Q_{\mathrm{EL}}-Q_{\mathrm{EL}} A$. Here, $P_{\mathrm{EL}}$ and $Q_{\mathrm{EL}}$ are the frequency-weighted grammians in (19) used in the combination method.

The new selection schemes provide a wide range of possibilities for combinations in choosing grammians, thus enlarging significantly the applicability of balancing-related approach to FWMR. For example, any combination of grammians $\left(P_{\mathrm{EL}}, Q_{\mathrm{V}}\right),\left(P_{\mathrm{V}}, Q_{\mathrm{EL}}\right)$, or $\left(P_{\mathrm{V}}, Q_{\mathrm{V}}\right)$, for all subunitary values of parameters $\alpha_{\mathrm{c}}$ and $\alpha_{\mathrm{o}}$ guarantees the stability of approximations for two-sided weighting. Note that the increase of computational cost associated with the use of the new selection schemes is practically negligible.

\section{Frequency-weighted controller reduction}

In this section we consider the application of balancingrelated FWMR techniques to solve the controller reduction problem (2) for the special weights defined in (3), (4), or (5). Assume that the open-loop system $\mathbf{G}=(A, B, C, D)$ has order $n$ and the stabilizing controller $\mathbf{K}=\left(A_{\mathrm{c}}, B_{\mathrm{c}}, C_{\mathrm{c}}, D_{\mathrm{c}}\right)$ has order $n_{\mathrm{c}}$. In the case of a stable controller, the computation of the frequency-weighted grammians $P_{\mathrm{E}}$ and $Q_{\mathrm{E}}$ apparently involves the solution of Lyapunov equations of order $n+2 n_{\mathrm{c}}$. Controller synthesis methods based on the LQG- or $H_{\infty}$-design methodologies lead typically to a controller order $n_{\mathrm{c}}=n$, so that, for the reduction of such controllers the solution of Lyapunov equations of order $3 n$ are apparently necessary. In what follows we show that it is always possible to solve Lyapunov equations of order at most $n+n_{\mathrm{c}}$ to compute the frequency-weighted controllability and observability grammians in controller reduction problems with the above special weights.

Since in general the controller can be unstable, only the stable part of the controller is reduced and a copy of the unstable part is kept in the reduced controller. Therefore, we assume a state-space representation of the controller with $A_{\mathrm{c}}$ already reduced to a block-diagonal form

$\mathbf{K}=\left[\begin{array}{c|c}A_{\mathrm{c}} & B_{\mathrm{c}} \\ \hline C_{\mathrm{c}} & D_{\mathrm{c}}\end{array}\right]=\left[\begin{array}{cc|c}A_{\mathrm{c} 1} & 0 & B_{\mathrm{c} 1} \\ 0 & A_{\mathrm{c} 2} & B_{\mathrm{c} 2} \\ \hline C_{\mathrm{c} 1} & C_{\mathrm{c} 2} & D_{\mathrm{c}}\end{array}\right]$,

where $\Lambda\left(A_{\mathrm{c} 1}\right) \subset C^{+}$and $\Lambda\left(A_{\mathrm{c} 2}\right) \subset C^{-}$. Here $C^{-}$denotes the open left half complex plane, while $C^{+}$denotes the complement of $C^{-}$in $C$. The above form corresponds to an additive decomposition of the controller TFM as $K=K_{\mathrm{u}}+K_{\mathrm{s}}$, where $\mathbf{K}_{\mathrm{u}}=\left(A_{\mathrm{c} 1}, B_{\mathrm{c} 1}, C_{\mathrm{c} 1}, 0\right)$ contains the unstable poles of $\mathbf{K}$ and $\mathbf{K}_{\mathrm{s}}=\left(A_{\mathrm{c} 2}, B_{\mathrm{c} 2}, C_{\mathrm{c} 2}, D_{\mathrm{c}}\right)$, of order $n^{-}$, contains the stable poles of $\mathbf{K}$.

For our developments, we build the state matrix of the realizations of the weights in (3), (4), or (5) in the form

$A_{\mathrm{w}}=\left[\begin{array}{cc}A-B D_{\mathrm{c}} R^{-1} C & B \tilde{R}^{-1} C_{\mathrm{c}} \\ -B_{\mathrm{c}} R^{-1} C & A_{\mathrm{c}}-B_{\mathrm{c}} R^{-1} D C_{\mathrm{c}}\end{array}\right]$,

where $R=I+D D_{\mathrm{c}}$ and $\tilde{R}=I+D_{\mathrm{c}} D$. Since the controller is stabilizing, $A_{\mathrm{w}}$ has all its eigenvalues in $C^{-}$.

The following theorem extends the result of Schelfhout and De Moor (1996) obtained for a stable controller stabilizing a strictly proper plant $(D=0)$.

Theorem 1. For a given $n$th order continuous-time system $\mathbf{G}=(A, B, C, D)$ assume that $\mathbf{K}=\left(A_{\mathrm{c}}, B_{\mathrm{c}}, C_{\mathrm{c}}, D_{\mathrm{c}}\right)$ is an $n_{\mathrm{c}}$ th order stabilizing controller with $I+D D_{\mathrm{c}}$ nonsingular and having a state-space realization of the form (22). Then, the 
frequency-weighted controllability and observability grammians, $P_{\mathrm{E}}$ and $Q_{\mathrm{E}}$, respectively, for Enns' method applied to frequency-weighting controller reduction problems with weights defined in (3), (4), or (5) can be computed by solving Lyapunov equations of order at most $n+n_{\mathrm{c}}$ as follows:

(1) For $W_{\mathrm{o}}=(I+G K)^{-1} G$ and $W_{\mathrm{i}}=I, P_{\mathrm{E}}$ satisfies

$$
A_{\mathrm{c} 2} P_{\mathrm{E}}+P_{\mathrm{E}} A_{\mathrm{c} 2}^{\mathrm{T}}+B_{\mathrm{c} 2} B_{\mathrm{c} 2}^{\mathrm{T}}=0
$$

and $Q_{\mathrm{E}}$ is the $n^{-} \times n^{-}$trailing block of $Q_{\mathrm{o}}$ satisfying

$$
A_{\mathrm{w}}^{\mathrm{T}} Q_{\mathrm{o}}+Q_{\mathrm{o}} A_{\mathrm{w}}+C_{\mathrm{o}}^{\mathrm{T}} C_{\mathrm{o}}=0
$$

with $C_{\mathrm{o}}=\left[-R^{-1} C-R^{-1} D C_{\mathrm{c}}\right]$.

(2) For $W_{\mathrm{o}}=I$ and $W_{\mathrm{i}}=G(I+G K)^{-1}, P_{\mathrm{E}}$ is the $n^{-} \times n^{-}$ trailing block of $P_{\mathrm{i}}$ satisfying

$$
A_{\mathrm{w}} P_{\mathrm{i}}+P_{\mathrm{i}} A_{\mathrm{w}}^{\mathrm{T}}+B_{\mathrm{i}} B_{\mathrm{i}}^{\mathrm{T}}=0
$$

with

$$
B_{\mathrm{i}}=\left[\begin{array}{c}
-B \tilde{R}^{-1} \\
B_{\mathrm{c}} D \tilde{R}^{-1}
\end{array}\right]
$$

and $Q_{\mathrm{E}}$ satisfies

$$
A_{\mathrm{c} 2}^{\mathrm{T}} Q_{\mathrm{E}}+Q_{\mathrm{E}} A_{\mathrm{c} 2}+C_{\mathrm{c} 2}^{\mathrm{T}} C_{\mathrm{c} 2}=0 .
$$

(3) For $W_{\mathrm{o}}=(I+G K)^{-1} G$ and $W_{\mathrm{i}}=(I+G K)^{-1}, P_{\mathrm{E}}$ is the $n^{-} \times n^{-}$trailing block of $P_{\mathrm{i}}$ satisfying (25) with

$$
B_{\mathrm{i}}=\left[\begin{array}{c}
B D_{\mathrm{c}} R^{-1} \\
B_{\mathrm{c}} R^{-1}
\end{array}\right]
$$

and $Q_{\mathrm{E}}$ is the $n^{-} \times n^{-}$trailing block of $Q_{\mathrm{o}}$ satisfying (24).

Proof. For details see (Varga \& Anderson, 2002).

By solving lower order Lyapunov equations, the achieved reduction of the computational effort can be significant for large order systems. For example, if $n_{\mathrm{c}}=n$, by solving $2 n$ order Lyapunov equations instead of $3 n$ order ones, the computation of one of grammians can be done $(3 / 2)^{3} \approx 3.4$ times faster.

If we try to apply the method of Lin and Chiu (1992), we get $P_{\mathrm{L}}=0$, and/or $Q_{\mathrm{L}}=0$, because pole-zero cancellations take place when forming $W_{\mathrm{o}} K_{\mathrm{s}}$ and/or $K_{\mathrm{s}} W_{\mathrm{i}}$. It follows that this method is not applicable for this class of controller reduction problems. Since the grammians for the proposed combination method are $P_{\mathrm{EL}}=\left(1-\alpha_{\mathrm{c}}^{2}\right) P_{\mathrm{E}}$ and $Q_{\mathrm{EL}}=\left(1-\alpha_{\mathrm{o}}^{2}\right) Q_{\mathrm{E}}$, this choice of grammians for subunitary $\alpha_{\mathrm{c}}$ and $\alpha_{\mathrm{o}}$ is practically the same as for Enns' method. To guarantee the stability of the reduced stable part of the controller, the choice $P_{\mathrm{V}}$ or $Q_{\mathrm{V}}$ corresponding to the modified combination method is appropriate.

Simplifications arise also in the case of a state-feedback and full-order observer based controller of the form

$\mathbf{K}=(A+B F+L C+L D F, L, F, 0)$.
The following result extends Lemma 1 of Liu et al. (1990) to the case of possibly unstable controllers.

Corollary 2. For a given $n$th order system $\mathbf{G}=(A, B, C, D)$ suppose $F$ is a state feedback gain and $L$ is a state estimator gain, such that $A+B F$ and $A+L C$ are stable. Then the frequency-weighted controllability and observability grammians for the method of Enns (1984) applied to the frequency-weighted controller reduction problems with weights defined in (3), (4), or (5) can be computed by solving Lyapunov equations of order at most $2 n$.

In the case of state feedback and observer based controllers important computational effort saving results if we further exploit the problem structure. In this case

$A_{\mathrm{w}}=\left[\begin{array}{cc}A & B F \\ -L C & A+B F+L C\end{array}\right]$

and this matrix can be put in an upper block diagonal form using the transformation matrix

$T=\left[\begin{array}{ll}I & 0 \\ I & I\end{array}\right]$.

We obtain the transformed matrices $\tilde{A}_{\mathrm{w}}:=T^{-1} A_{\mathrm{w}} T$, $\tilde{B}_{\mathrm{i}}:=T^{-1} B_{\mathrm{i}}$, and $\tilde{C}_{\mathrm{o}}:=C_{\mathrm{o}} T$, where

$\tilde{A}_{\mathrm{w}}=\left[\begin{array}{cc}A+B F & B F \\ 0 & A+L C\end{array}\right]$.

If $\tilde{P}_{\mathrm{i}}$ and $\tilde{Q}_{\mathrm{o}}$ satisfy

$$
\begin{aligned}
& \tilde{A}_{\mathrm{w}} \tilde{P}_{\mathrm{i}}+\tilde{P}_{\mathrm{i}} \tilde{A}_{\mathrm{w}}^{\mathrm{T}}+\tilde{B}_{\mathrm{i}} \tilde{B}_{\mathrm{i}}^{\mathrm{T}}=0 \\
& \tilde{A}_{\mathrm{w}}^{\mathrm{T}} \tilde{Q}_{\mathrm{o}}+\tilde{Q}_{\mathrm{o}} \tilde{A}_{\mathrm{w}}+\tilde{C}_{\mathrm{o}}^{\mathrm{T}} \tilde{C}_{\mathrm{o}}=0
\end{aligned}
$$

then $P_{\mathrm{i}}$ in (25) and $Q_{\mathrm{o}}$ in (24) are given by $P_{\mathrm{i}}=T \tilde{P}_{\mathrm{i}} T^{\mathrm{T}}$ and $Q_{\mathrm{o}}=T^{-\mathrm{T}} \tilde{Q}_{\mathrm{o}} T^{-1}$, respectively. The computational saving arises from the need to reduce $A_{\mathrm{w}}$ to a real Schur form (RSF) when solving the Lyapunov equations (24) and (25). Instead of reducing the $2 n \times 2 n$ matrix $A_{\mathrm{w}}$, we can reduce two $n \times n$ matrices $A+B F$ and $A+L C$ to obtain $\tilde{A}_{\text {w }}$ in a RSF. This means a 4 times speedup of computations for this step.

\section{Accuracy-enhancing techniques}

\subsection{Square-root and balancing-free techniques}

The emphasis on improving the accuracy of computations in model reduction has led to the so-called model reduction algorithms with enhanced accuracy. Several techniques can be employed to enhance the accuracy of computed reduced models. The square-root (SR) method, introduced by Tombs and Postlethwaite (1987), determines the truncation matrices $L$ and $T$ according to (11). These matrices are 
then used to compute the reduced order model via (8). Thus, the computation of the matrices of the reduced model can be done entirely on the basis of the Cholesky factors $S$ and $R$ of the positive semidefinite grammians, satisfying $P=S S^{\mathrm{T}}$ and $Q=R^{\mathrm{T}} R$. This approach is numerically appealing because the upper triangular Cholesky factors $R$ and $S$ can be computed directly by solving the Lyapunov equations satisfied by the grammians (Hammarling, 1982). For well-equilibrated systems this approach is usually numerically very accurate. The alternative to compute first the grammians and then their Cholesky factors is numerically unsatisfactory, because roundoff errors can lead to the loss of positive definiteness of the computed grammians and thus make the computation of factors numerically unreliable.

Potential accuracy losses can be induced in the reduced model if either of the truncation matrices $L$ or $T$ is numerically nearly rank deficient. Recall that $T$ and $L$ in the SR method, are the first $r$ columns and first $r$ rows of the balancing transformation matrix $Z$ in (7) and its inverse, respectively. If the original system is highly unbalanced, $Z$ can become very ill-conditioned and thus the accuracy of computations can be adversely affected. To avoid ill-conditioned truncation matrices, a balancing-free (BF) approach has been proposed by Safonov and Chiang (1989) in which $L$ and $T$ are always well-conditioned if a sufficient gap exists between the singular values $\sigma_{r}$ and $\sigma_{r+1}$ (i.e., $\sigma_{r} \gg \sigma_{r+1}$ ). The truncation matrices are computed from two matrices whose orthogonal columns span bases for the right and left eigenspaces of the product $P Q$ corresponding to the first $r$ largest eigenvalues $\sigma_{1}^{2}, \ldots, \sigma_{r}^{2}$. However, because of the need to compute explicitly $P$ and $Q$ as well as their product, this approach is usually less accurate for moderately ill-balanced systems than the $\mathbf{S R}$ approach.

A balancing-free square-root (BFSR) algorithm which combines the advantages of the $\mathbf{B F}$ and $\mathbf{S R}$ approaches has been proposed by Varga (1991b). Among several ways to choose $L$ and $T$, a convenient choice is

$L=\left(Y^{\mathrm{T}} X\right)^{-1} Y^{\mathrm{T}}, \quad T=X$,

where $X$ and $Y$ are $n \times r$ matrices with orthonormal columns computed from two QR decompositions

$S V_{1}=X W, \quad R^{\mathrm{T}} U_{1}=Y Z$

with $W$ and $Z$ non-singular and upper-triangular. Note that by this choice, the reduced model computed by the BT method is related by a system similarity transformation to the reduced model obtained by the SR method. The above choice of truncation matrices involves two QR-decompositions and the inversion of an $r \times r$ matrix and is cheaper than the choice suggested by Safonov and Chiang (1989) involving, instead inversion, the SVD of the product $Y^{\mathrm{T}} X$. The accuracy of the BFSR algorithm is usually better than either of SR or BF approaches.

The SPA method can be used directly on a balanced minimal order realization of the original system computed with the SR method. A BFSR method to compute SPAs has been proposed by Varga (1991a). The essence of this method is to construct the truncation matrices $L$ and $T$ such that the system $(L A T, L B, C T, D)$ is a minimal realization of the original system and the product of corresponding grammians is block-diagonal, allowing thus the application of the SPA formulas.

The effectiveness of the SR or BFSR techniques depends mostly on the accuracy of the computed Cholesky factors of the grammians. Having the Cholesky factors for the frequency-weighted grammians, we can compute in a numerically reliable way the truncation matrices $L$ and $T$ using either the SR or BFSR technique. In what follows, we develop explicit formulas to compute the Cholesky factors of the frequency-weighted grammians for the balancing-related frequency-weighted model and controller reduction. With these formulas, the new SR or BFSR formulations of both the FWBT and FWSPA methods are entirely satisfactory from a numerical point of view and can serve as a basis for robust software implementations of the frequency-weighted balancing-related approach.

\subsection{Square-root techniques for FWMR}

In this section we show how square-root formulas can be employed to compute the frequency-weighted grammians for the specific choices described in the Section 2. Assume $\bar{S}$ and $\bar{R}$ are the Cholesky factors of $\bar{P}$ and $\bar{Q}$ in (14), respectively, satisfying $\bar{P}=\bar{S} \bar{S}^{\mathrm{T}}$ and $\bar{Q}=\bar{R}^{\mathrm{T}} \bar{R}$. These factors can be computed with the method of Hammarling (1982) by solving directly for the Cholesky factors the Lyapunov equations

$$
\begin{aligned}
& \bar{A}_{\mathrm{i}} \bar{P}+\bar{P} \bar{A}_{\mathrm{i}}^{\mathrm{T}}+\bar{B}_{\mathrm{i}} \bar{B}_{\mathrm{i}}^{\mathrm{T}}=0, \\
& \bar{A}_{\mathrm{o}}^{\mathrm{T}} \bar{Q}+\bar{Q} \bar{A}_{\mathrm{o}}+\bar{C}_{\mathrm{o}}^{\mathrm{T}} \bar{C}_{\mathrm{o}}=0 .
\end{aligned}
$$

If we partition $\bar{S}$ and $\bar{R}$ as

$\bar{S}=\left[\begin{array}{cc}S_{11} & S_{12} \\ 0 & S_{22}\end{array}\right], \quad \bar{R}=\left[\begin{array}{cc}R_{11} & R_{12} \\ 0 & R_{22}\end{array}\right]$

then the Cholesky factors of $P_{\mathrm{E}}=S_{\mathrm{E}} S_{\mathrm{E}}^{\mathrm{T}}$ and $Q_{\mathrm{E}}=R_{\mathrm{E}}^{\mathrm{T}} R_{\mathrm{E}}$ corresponding to Enns' method are given by

$$
\begin{aligned}
& S_{\mathrm{E}} S_{\mathrm{E}}^{\mathrm{T}}=S_{11} S_{11}^{\mathrm{T}}+S_{12} S_{12}^{\mathrm{T}}=\left[\begin{array}{ll}
S_{11} & S_{12}
\end{array}\right]\left[\begin{array}{c}
S_{11}^{\mathrm{T}} \\
S_{12}^{\mathrm{T}}
\end{array}\right], \\
& R_{\mathrm{E}}^{\mathrm{T}} R_{\mathrm{E}}=R_{22}^{\mathrm{T}} R_{22}+R_{12}^{\mathrm{T}} R_{12}=\left[\begin{array}{ll}
R_{22}^{\mathrm{T}} & R_{12}^{\mathrm{T}}
\end{array}\right]\left[\begin{array}{c}
R_{22} \\
R_{12}
\end{array}\right] .
\end{aligned}
$$

Thus, to compute $S_{\mathrm{E}}$ the RQ-factorization of the matrix $\left[\begin{array}{ll}S_{11} & S_{12}\end{array}\right]$ must be performed, while for computing $R_{\mathrm{E}}$ the QR-factorization of $\left[\begin{array}{ll}R_{22}^{\mathrm{T}} & R_{12}^{\mathrm{T}}\end{array}\right]^{\mathrm{T}}$ must be performed. Both these factorizations can be computed using factorization updating techniques (Gill, Golub, Murray, \& Saunders, 1974) 
which fully exploit the upper triangular shapes of $S_{11}$ and $R_{22}$.

The Cholesky factors of $P_{\mathrm{L}}=S_{\mathrm{L}} S_{\mathrm{L}}^{\mathrm{T}}$ and $Q_{\mathrm{L}}=R_{\mathrm{L}}^{\mathrm{T}} R_{\mathrm{L}}$ for the method of Lin and Chiu (1992) are

$S_{\mathrm{L}}=S_{11}, \quad R_{\mathrm{L}}=R_{22}$

Thus, by working directly with the Cholesky factors we completely avoid the matrix inversions necessary when forming $P_{\mathrm{L}}$ and $Q_{\mathrm{L}}$ directly from (16). Therefore, no difficulties are expected to be encountered when $P_{22}=S_{22} S_{22}^{\mathrm{T}}$ or $Q_{11}=R_{11}^{\mathrm{T}} R_{11}$ are ill-conditioned or even exactly singular (i.e., the state space representations of $W_{\mathrm{i}}$ and/or $W_{\mathrm{o}}$ are not minimal).

For the combination method, the Cholesky factors of $P_{\mathrm{EL}}=S_{\mathrm{EL}} S_{\mathrm{EL}}^{\mathrm{T}}$ and $Q_{\mathrm{EL}}=R_{\mathrm{EL}}^{\mathrm{T}} R_{\mathrm{EL}}$ are given by

$S_{\mathrm{EL}} S_{\mathrm{EL}}^{\mathrm{T}}=S_{11} S_{11}^{\mathrm{T}}+\left(1-\alpha_{\mathrm{c}}^{2}\right) S_{12} S_{12}^{\mathrm{T}}$,

$R_{\mathrm{EL}}^{\mathrm{T}} S_{\mathrm{EL}}=R_{22}^{\mathrm{T}} R_{22}+\left(1-\alpha_{\mathrm{o}}^{2}\right) R_{12}^{\mathrm{T}} R_{12}$

and can be computed from the RQ-decomposition of $\left[\begin{array}{lll}S_{11} & \sqrt{1-\alpha_{\mathrm{c}}^{2}} S_{12}\end{array}\right]$ and QR-decomposition of $\left[R_{22}^{\mathrm{T}} \sqrt{1-\alpha_{0}^{2}} R_{12}^{\mathrm{T}}\right]^{\mathrm{T}}$. For the modified combination method, the Cholesky factors of $P_{\mathrm{V}}=S_{\mathrm{V}} S_{\mathrm{V}}^{\mathrm{T}}$ and $Q_{\mathrm{V}}=R_{\mathrm{V}}^{\mathrm{T}} R_{\mathrm{V}}$ result by solving (21) directly for these factors using the algorithm of Hammarling (1982).

The solution of the Lyapunov equations (28) involves the orthogonal reduction of both $\bar{A}_{\mathrm{i}}$ and $\bar{A}_{\mathrm{o}}$ to RSF. This reduction can automatically be achieved by performing appropriate orthogonal similarity transformations on the state-space realizations $\mathbf{G}, \mathbf{W}_{\mathrm{i}}$ and $\mathbf{W}_{\mathrm{o}}$ to reduce independently the state matrices $A, A_{\mathrm{i}}$ and $A_{\mathrm{o}}$ to RSF.

\subsection{Square-root techniques for controller reduction}

We can employ the method of Hammarling (1982) to solve (25) and (24) directly for the Cholesky factors $S_{\mathrm{i}}$ of $P_{\mathrm{i}}=S_{\mathrm{i}} S_{\mathrm{i}}^{\mathrm{T}}$ and $R_{\mathrm{o}}$ of $Q_{\mathrm{o}}=R_{\mathrm{o}}^{\mathrm{T}} R_{\mathrm{o}}$, respectively. In the case of an unstable controller, we assume a state space realization of $\mathbf{K}$ as in (22) with the $n^{-} \times n^{-}$matrix $A_{\mathrm{c} 2}$ containing the stable eigenvalues of $A_{\mathrm{c}}$. If we partition $S_{\mathrm{i}}$ and $R_{\mathrm{o}}$ in the form

$S_{\mathrm{i}}=\left[\begin{array}{cc}S_{11} & S_{12} \\ 0 & S_{22}\end{array}\right], \quad R_{\mathrm{o}}=\left[\begin{array}{cc}R_{11} & R_{12} \\ 0 & R_{22}\end{array}\right]$,

where both $S_{22}$ and $R_{22}$ are $n^{-} \times n^{-}$, then the Cholesky factor of the trailing block of $P_{\mathrm{i}}$ in (25) corresponding to the stable part of $\mathbf{K}$ is simply $S_{\mathrm{E}}=S_{22}$, while the Cholesky factor $R_{\mathrm{E}}$ of the trailing block of $Q_{\mathrm{o}}$ in (24) satisfies $R_{\mathrm{E}}^{\mathrm{T}} R_{\mathrm{E}}=R_{22}^{\mathrm{T}} R_{22}+$ $R_{12}^{\mathrm{T}} R_{12}$. Thus the computation of $R_{\mathrm{E}}$ involves an additional QR-decomposition of $\left[\begin{array}{ll}R_{22}^{\mathrm{T}} & R_{12}^{\mathrm{T}}\end{array}\right]^{\mathrm{T}}$ and can be computed using standard updating techniques (Gill et al., 1974). Updating can be avoided in the case of one-sided weight $W_{\mathrm{o}}=$ $(I+G K)^{-1} G$, by using alternative state-space realizations of
$\mathbf{W}_{\mathrm{o}}$ and $\mathbf{K}$. For details, see (Varga \& Anderson, 2002). Still in the case of two-sided weighting with $W_{\mathrm{o}}=(I+G K)^{-1} G$ and $W_{\mathrm{i}}=(I+G K)^{-1}$ we prefer the approach of the Theorem 1 with $\mathbf{W}_{\mathrm{i}}$ and $\mathbf{W}_{\mathrm{o}}$ sharing the same state matrix $A_{\mathrm{w}}$, because the computation of both grammians can be done with a single reduction of this $\left(n+n_{\mathrm{c}}\right) \times\left(n+n_{\mathrm{c}}\right)$ matrix to the RSF. In this case the cost to compute the two grammians is practically the same as for one grammian.

For a state-feedback and full-observer based controller, let $\tilde{S}_{\mathrm{i}}$ be the Cholesky factor of $\tilde{P}_{\mathrm{i}}$ in (27) partitioned as

$\tilde{S}_{\mathrm{i}}=\left[\begin{array}{cc}\tilde{S}_{11} & \tilde{S}_{12} \\ 0 & \tilde{S}_{22}\end{array}\right]$.

The $n^{-} \times n^{-}$Cholesky factor $S_{\mathrm{E}}$ corresponding to the trailing $n^{-} \times n^{-}$part of $P_{\mathrm{i}}$ is the trailing $n^{-} \times n^{-}$block of an upper triangular matrix $\hat{S}_{22}$ which satisfies

$\hat{S}_{22} \hat{S}_{22}^{\mathrm{T}}=\tilde{S}_{11} \tilde{S}_{11}^{\mathrm{T}}+\left(\tilde{S}_{12}+\tilde{S}_{22}\right)\left(\tilde{S}_{12}+\tilde{S}_{22}\right)^{\mathrm{T}}$

$\hat{S}_{22}$ can be computed easily from the RQ-decomposition of

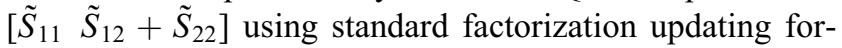
mulas (Gill et al., 1974). No difference appears in the computation of the Cholesky factor $R_{\mathrm{E}}$.

\section{Numerical example}

We present an example which is only intended to illustrate the proposed enhancements of Enns' method. This is the fourth-order model used in Example 1 of Wang et al. (1999) with the frequency weights

$\mathbf{W}_{\mathrm{i}}=\mathbf{W}_{\mathrm{o}}=\left(-4.5 I_{2}, 3 I_{2}, 1.5 I_{2}, I_{2}\right)$.

We computed approximations $\mathbf{G}_{r}$ of orders $r=1,2$ and 3 using the combination method for values of $\alpha=\alpha_{c}=$ $\alpha_{0} 0,0.5$ and 1. Recall that $\alpha=0$ corresponds to Enns' method while $\alpha=1$ corresponds to the method of Lin and Chiu (1992). In Table 1 we present the resulting weighted approximation error $\left\|W_{\mathrm{o}}\left(G-G_{r}\right) W_{\mathrm{i}}\right\|_{\infty}$ for the FWBT and FWSPA methods. For comparison purposes, we have also added in the last column the results reported for the method of Wang et al. (1999) (WSL).

The modified combination method, with $\alpha=0$ and the pair $\left(P_{\mathrm{V}}, Q_{\mathrm{V}}\right)$ computed as in $(21)$, leads for both FWBT and FWSPA to almost the same errors as those in Table 1. In all cases the errors are smaller than those for the method of Wang et al. (1999). The FWSPA method for $\alpha=0$ (see values in bold face) behaves uniformly better on this example than all other approaches. We postulate that the good behaviour is probably due to the fact that, by forcing a zero error in the steady-state gain, lower error results also in the low frequency domain which partly overlaps with the frequency region where the weights are most active. 
Table 1

Weighted errors for the FWBT, FWSPA and WSL methods

\begin{tabular}{|c|c|c|c|c|c|c|c|}
\hline \multirow[b]{2}{*}{$r$} & \multicolumn{3}{|c|}{ FWBT } & \multicolumn{3}{|c|}{ FWSPA } & \multirow[t]{2}{*}{ WSL } \\
\hline & $\alpha=0$ & $\alpha=0.5$ & $\alpha=1$ & $\alpha=0$ & $\alpha=0.5$ & $\alpha=1$ & \\
\hline 1 & 2.112 & 2.116 & 2.566 & 1.405 & 1.495 & 2.035 & 2.121 \\
\hline 2 & 0.265 & 0.261 & 0.560 & 0.250 & 0.256 & 0.687 & 0.272 \\
\hline 3 & 0.112 & 0.110 & 0.164 & 0.065 & 0.069 & 0.121 & 0.115 \\
\hline
\end{tabular}

\section{Conclusions}

We proposed three enhancements of Enns' method which substantially enlarge the applicability of balancing related FWMR approaches: (1) the FWSPA approach; (2) new choices of the frequency-weighted controllability and observability grammians ensuring the stability of approximations for two-sided frequency weighting; and (3) increased numerical accuracy of overall computations by extending the balancing-free square-root techniques to the frequency-weighted balancing-related model reduction. The proposed enhancements can be immediately applied to solve frequency-weighted controller reduction problems as well. For a special class of stability/performance preserving controller reduction problems, we have shown that by applying the FWMR approach, a significant reduction of the computational burden can be achieved by exploiting the particular structure of the problem. All these results apply in the discrete-time case as well (Varga \& Anderson, 2001, 2002). For the newly developed model and controller reduction methods, robust numerical software has been implemented by Varga (2002) as part of the freely available Fortran 77 library SLICOT. To facilitate the usage of these tools, easy-to-use, flexible and user friendly interfaces have been developed to integrate them in MATLAB.

\section{References}

Anderson, B. D. O., \& Liu, Y. (1989). Controller reduction: Concepts and approaches. IEEE Transactions of Automatic Control, 34, 802-812.

Enns, D. (1984). Model reduction for control systems design. Ph.D. thesis. Dept. Aeronaut. Astronaut., Stanford Univ., Stanford, CA.

Gill, P. E., Golub, G. H., Murray, W., \& Saunders, M. A. (1974). Methods for modifying matrix factorizations. Mathematics of Computation, 28, 505-535.

Hammarling, S. J. (1982). Numerical solution of the stable, non-negative definite Lyapunov equation. IMA Journal of Numerical Analysis, 2, 303-323.

Lin, C. -A., \& Chiu, T. -Y. (1992). Model reduction via frequency weighted balanced realization. CONTROL-Theory and Advanced Technology, 8, 341-351.

Liu, Y., \& Anderson, B. D. O. (1989). Singular perturbation approximation of balanced systems. International Journal of Control, 50, 1379-1405.

Liu, Y., Anderson, B. D. O., \& Ly, U. L. (1990). Coprime factorization controller reduction with Bezout identity induced frequency weighting. Automatica, 26, 233-249.
Moore, B. C. (1981). Principal component analysis in linear system: Controllability, observability and model reduction. IEEE Transactions on Automatic Control, 26, 17-32.

Pernebo, L., \& Silverman, L. M. (1982). Model reduction via balanced state space representations. IEEE Transactions of Automatic Control, 27, 382-387.

Safonov, M. G., \& Chiang, R. Y. (1989). A Schur method for balancedtruncation model reduction. IEEE Transactions of Automatic Control, $34,729-733$.

Schelfhout, G., \& De Moor, B. (1996). A note on closed-loop balanced truncation. IEEE Transactions of Automatic Control, 41, 1498-1500.

Tombs, M. S., \& Postlethwaite, I. (1987). Truncated balanced realization of a stable non-minimal state-space system. International Journal of Control, 46, 1319-1330.

Varga, A. (1991a). Balancing-free square-root algorithm for computing singular perturbation approximations (pp. 1062-1065). Proceedings of the 30th IEEE CDC. Brighton, UK.

Varga, A. (1991b). Efficient minimal realization procedure based on balancing. In A. El Moudni, P. Borne, \& S. G. Tzafestas (Eds.), Preprints of IMACS Symposium on Modelling and Control of Technological Systems, Lille, France, Vol. 2 (pp. 42-47).

Varga, A. (2001). Model reduction software in the SLICOT library. In B. N. Datta, (Ed.), Applied and computational control, signals and circuits. Kluwer International Series in Engineering and Computer Science, Vol. 629 (pp. 239-282). Boston: Kluwer Academic Publishers.

Varga, A. (2002). Numerical software in SLICOT for low order controller design. In Proceedings of the CACSD'2002 Symposium. Glasgow, UK.

Varga, A., \& Anderson, B. D. O. (2001). Square-root balancing-free methods for the frequency-weighted balancing related model reduction. Proceedings of the CDC'2001 (pp. 3659-3664). Orlando, FL.

Varga, A., \& Anderson, B. D. O. (2002). Frequency-weighted balancing related controller reduction. Proceedings of the IFAC'2002 congress. Barcelona, Spain.

Wang, G., Sreeram, V., \& Liu, W. Q. (1999). A new frequency-weighted balanced truncation method and error bound. IEEE Transactions of Automatic Control, 44, 1734-1737.

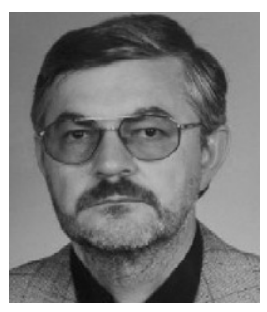

Andras Varga received the diploma in control engineering in 1974 and the Ph.D. degree in electrical engineering in 1981, both from the University "Politechnica" of Bucharest (Romania). From 1974 to 1993 he has held various research positions at the Institute of Informatics Bucharest and at the Ruhr-University of Bochum. From 1990 to 1992 he worked at the Ruhr-University of Bochum as visiting research fellow in the framework of a fellowship award of the Alexander von Humboldt Foundation. Since 1993, he has been at the German Aerospace Center (DLR) in Oberpfaffenhofen, where he is currently a Senior Scientist. He was a visiting scholar at the Kyoto University (1994), California Institute of Technology (2000), Australian National University (2000), University of Hong Kong (2000), and University of Umea (2002). Dr. Varga's main 
research interests include the numerical methods for linear systems analysis and design (with special emphasis on model and controller reduction, descriptor systems, periodic systems), and robust numerical software for computer aided control system design (CACSD). He coauthored three books and coedited one book, published over 40 papers in refereed journals, and has over 90 conference publications. Dr. Varga is Fellow of IEEE and served as Associate Editor for the IEEE Transactions on Automatic Control between 1997 and 1999. He was the Program Chairman of the 1999 Symposium on CACSD (Hawaii) and the General Chair for the 2000 Symposium on CACSD (Anchorage, Alaska). From 2000, he is the Chairman of the Technical Committee on CACSD within the IEEE Control Systems Society. For $2002-2003$ he was a nominated member of the Board of Governors of the IEEE Control Systems Society.

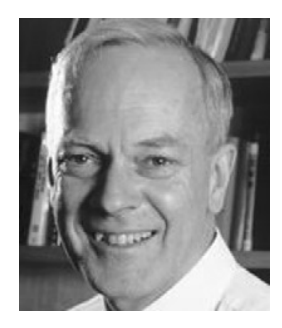

Brian Anderson was born in Sydney, Australia. He took his undergraduate degrees in Mathematics and Electrical Engineering at Sydney University, and his doctoral degree in Electrical Engineering at Stanford University in 1966. Professor Anderson is designate CEO, National ICT Australia. He worked in industry in the United States and at Stanford University before serving as Professor of Electrical Engineering at the University of Newcastle, Australia from 1967 through 1981. At that time, he took up a post as Professor and Head of the Department of Systems
Engineering, at the Australian National University in Canberra, where he was Director of the Research School of Information Sciences and Engineering from 1994-2002. He has held many visiting appointments in the United States, Asia and Europe, including the University of California (Berkeley), Stanford University, Tokyo Institute of Technology, and the Swiss Federal Institute of Technology. Professor Anderson has served as a member of a number of government bodies, including the Prime Minister's Science, Engineering and Innovation Council. He is also a member of the Board of Cochlear Limited, one of the world's major suppliers of cochlear implants. He is a Fellow of his own country's Academy of Science and Academy of Technological Sciences and Engineering, the Institute of Electrical and Electronic Engineers, and an Honorary Fellow of the Institution of Engineers, Australia. In 1989, he became a Fellow of the Royal Society and in 2002 a Foreign Associate of the US National Academy of Engineering. He holds honorary doctorates from the Universite Catholique de Louvain, Swiss Federal Institute of Technology (Zurich) and the Universities of Sydney, Melbourne and New South Wales. In 1998 he was elected President of the Australian Academy of Science for a four year term. Professor Anderson received the Quazza Medal at the IFAC 14th World Congress, Beijing, China in 1999 and an Automatica Prize Paper Award at the same time. He has held a number of offices in IFAC, including the Presidency for 1990-1993. 